Supporting Information

\title{
Different Effect of the Additional
}

\section{Electron-Withdrawing Cyano Group in Different Conjugation Bridge: The Adjusted Molecular} Energy Levels and Largely Improved Photovoltaic Performance

Huiyang Li ${ }^{a}$ Manman Fang, ${ }^{a}$ Yingqin Hou, ${ }^{a}$ Runli Tang, ${ }^{a}$ Yizhou Yang,,${ }^{a}$ Cheng Zhong, ${ }^{* a}$ Qianqian $L i{ }^{* a}$ and Zhen $L i{ }^{* a}$

${ }^{a}$ Department of Chemistry, Hubei Key Lab on Organic and Polymeric Opto-Electronic Materials, Wuhan University, Wuhan 430072, China.

Corresponding author. Phone: 86-27-68755363; Fax: 86-27-68756757; E-mail:

qianqian-alinda@163.com (Q. Li); zhongcheng@whu.edu.cn (C. Zhong); lizhen@whu.edu.cn (Z. Li). 


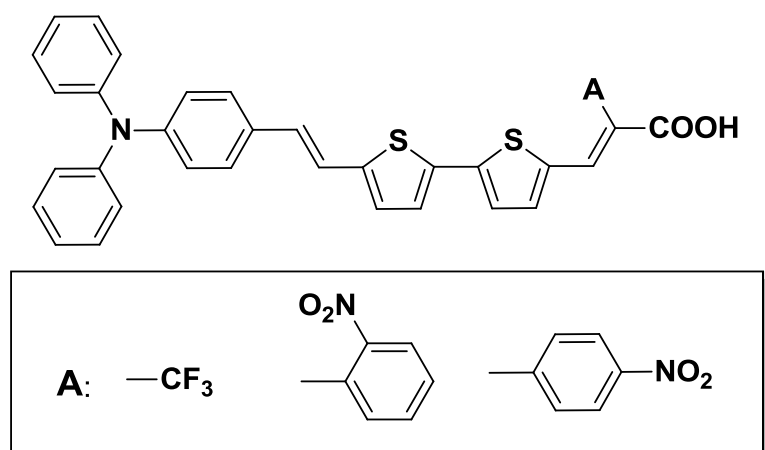

Chart S1 Organic dyes with different electron acceptors.
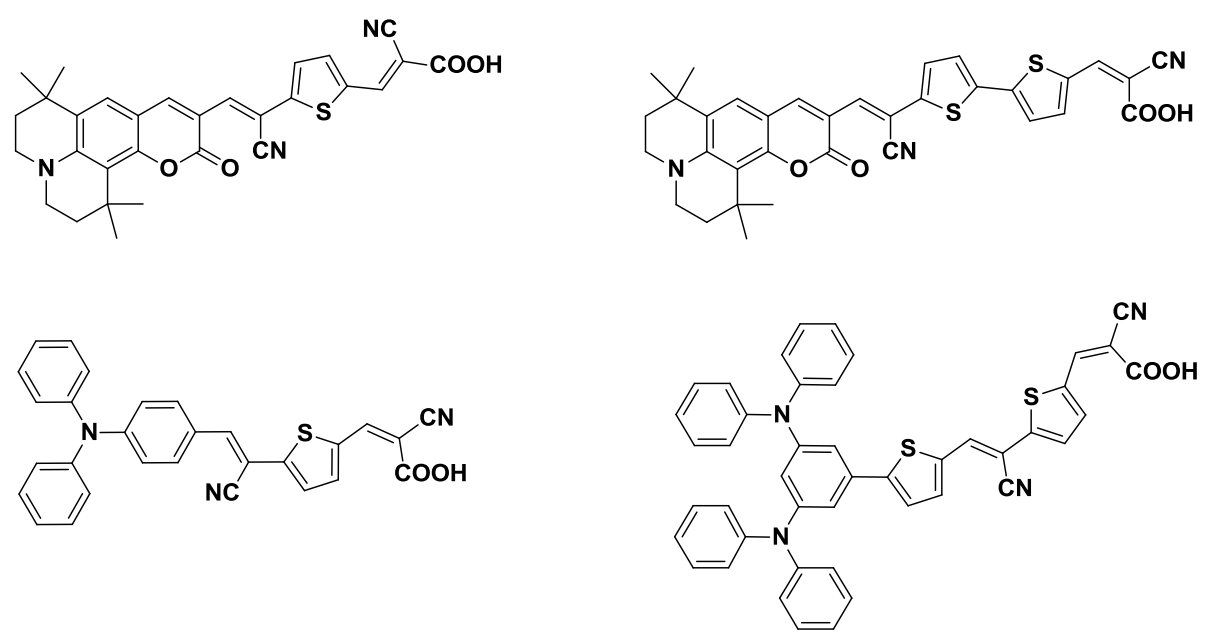

Chart S2 The structures of organic dyes bearing CN unit in thiophene-based conjugated bridge. 


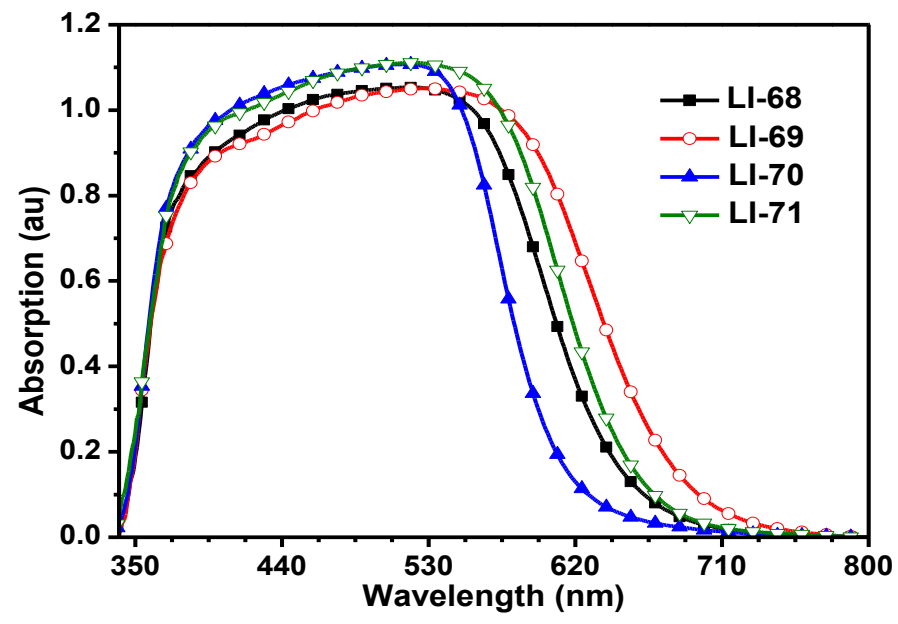

Figure S1 UV-vis spectra of the sensitizers on $\mathrm{TiO}_{2}$ films $(4 \mu \mathrm{m})$.

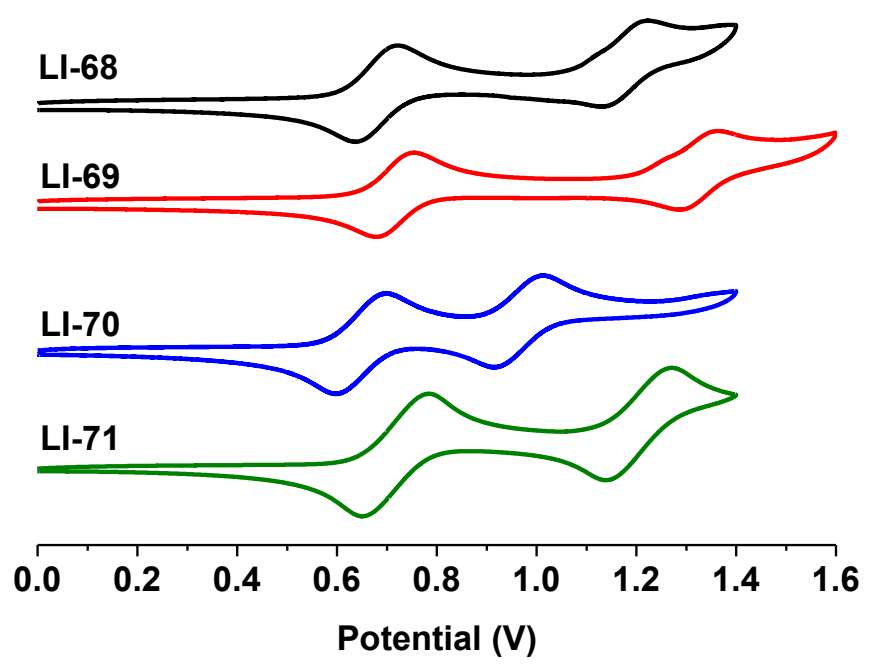

Figure S2 Cyclic voltammograms of the sensitizers in $\mathrm{CH}_{2} \mathrm{Cl}_{2}$. 

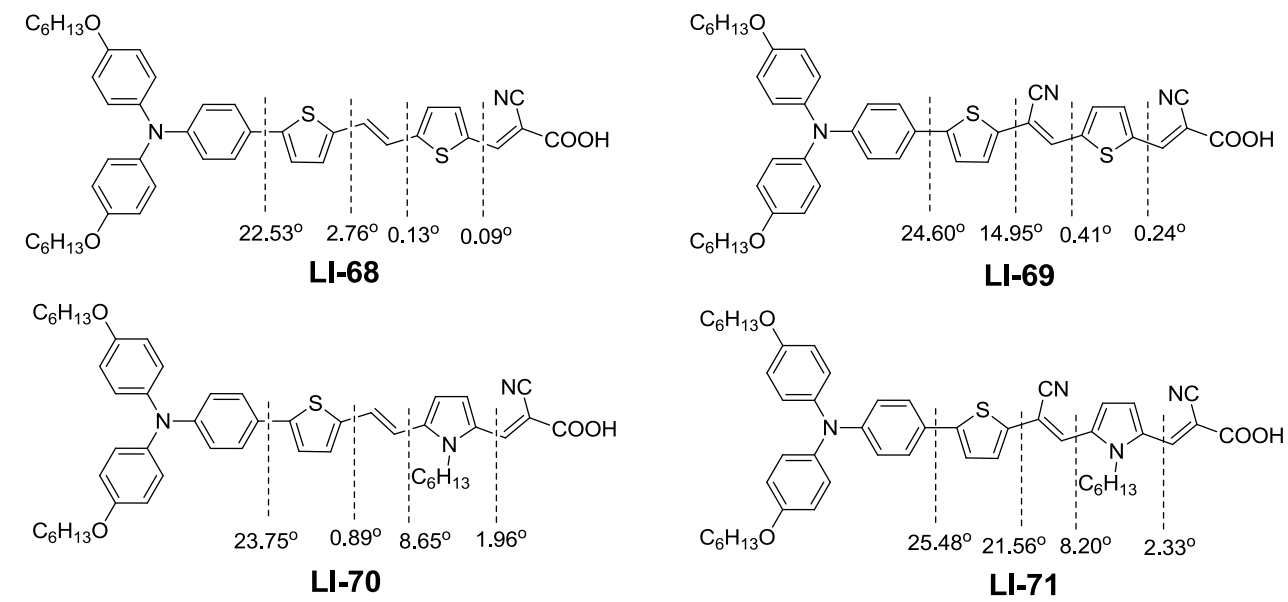

Figure S3 Calculated dihedral angles between the adjacent units.

Table S1 The difference values of the Mullikan charges from ground state to excited state

\begin{tabular}{lllllll}
\hline & & $\mathrm{D}$ & $\mathrm{B} 1$ & $\mathrm{~B} 2$ & $\mathrm{~B} 3$ & $\mathrm{~A}$ \\
\hline LI-68 & $\mathrm{S}_{0} \rightarrow \mathrm{S}_{1}$ & 0.415 & -0.074 & -0.057 & -0.148 & -0.204 \\
LI-69 & $\mathrm{S}_{0} \rightarrow \mathrm{S}_{1}$ & 0.412 & -0.071 & -0.120 & -0.118 & -0.175 \\
LI-70 & $\mathrm{S}_{0} \rightarrow \mathrm{S}_{1}$ & 0.409 & -0.080 & -0.075 & -0.111 & -0.209 \\
LI-71 & $\mathrm{S}_{0} \rightarrow \mathrm{S}_{1}$ & 0.452 & -0.059 & -0.171 & -0.095 & -0.203 \\
\hline
\end{tabular}

Table S2 Photovoltaic performance of DSCs by different fabrication method ${ }^{a}$

\begin{tabular}{|c|c|c|c|c|c|}
\hline sensitizers & $C D C A(\mathrm{mM})$ & $J_{S C}\left(\mathrm{~mA} \mathrm{~cm}^{-2}\right)$ & $V_{O C}(\mathrm{~V})$ & $F F$ & $\eta(\%)$ \\
\hline \multirow{2}{*}{ LI-68 } & 0 & $13.05 \pm 0.30$ & $0.62 \pm 0.01$ & $0.70 \pm 0.02$ & $5.71 \pm 0.27$ \\
\cline { 2 - 6 } & 5 & $14.14 \pm 0.48$ & $0.64 \pm 0.01$ & $0.71 \pm 0.02$ & $6.40 \pm 0.06$ \\
\hline \multirow{3}{*}{ LI-69 } & 0 & $5.31 \pm 0.34$ & $0.52 \pm 0.01$ & $0.74 \pm 0.01$ & $2.06 \pm 0.13$ \\
\cline { 2 - 6 } & 5 & $7.24 \pm 0.21$ & $0.53 \pm 0.01$ & $0.72 \pm 0.01$ & $2.76 \pm 0.11$ \\
\hline \multirow{2}{*}{ LI-70 } & 0 & $13.59 \pm 0.36$ & $0.63 \pm 0.01$ & $0.71 \pm 0.01$ & $6.05 \pm 0.14$ \\
\cline { 2 - 6 } & 5 & $14.35 \pm 0.35$ & $0.64 \pm 0.01$ & $0.69 \pm 0.01$ & $6.41 \pm 0.12$ \\
\hline \multirow{2}{*}{ LI-71 } & 0 & $12.41 \pm 0.09$ & $0.66 \pm 0.01$ & $0.77 \pm 0.01$ & $6.34 \pm 0.12$ \\
\cline { 2 - 6 } & 5 & $15.21 \pm 0.21$ & $0.66 \pm 0.01$ & $0.77 \pm 0.01$ & $7.79 \pm 0.26$ \\
\hline
\end{tabular}

${ }^{a}$ Prior to the deposition of the $\mathrm{TiO}_{2}$ paste, the cleaned conducting glass substrates (FTO, $4 \mathrm{~mm}$ thickness, $\sim 9 \mathrm{ohms} / \mathrm{sq}$ ) were immersed in a solution of $\mathrm{TiCl}_{4}(40 \mathrm{mM})$ at $70{ }^{\circ} \mathrm{C}$ for $30 \mathrm{~min}$, washed with water and ethanol and then dried. The photoanodes (thickness $18 \mathrm{~mm}$; area $\left.0.25 \mathrm{~cm}^{2}\right)$ were prepared using the screen printing technique containing a $15 \mu \mathrm{m}$ layer of mesoporous $\mathrm{TiO}_{2}(18 \mathrm{NR}-\mathrm{T}$, Dyesol) and a $3 \mu \mathrm{m}$ scatter layer (18NR-AO, Dyesol). The $\mathrm{TiO}_{2}$ electrodes were gradually heated under airflow at $325{ }^{\circ} \mathrm{C}$ for $5 \mathrm{~min}, 375{ }^{\circ} \mathrm{C}$ for $5 \mathrm{~min}, 450{ }^{\circ} \mathrm{C}$ for $15 \mathrm{~min}$ and $500{ }^{\circ} \mathrm{C}$ for $1 \mathrm{~h}$. After the film was cooled to room temperature, they were immersed again in a solution of TiCl $4(40 \mathrm{mM})$ at $70{ }^{\circ} \mathrm{C}$ for $30 \mathrm{~min}$, washed with water and ethanol and then dried. The electrodes were annealed at $500{ }^{\circ} \mathrm{C}$ for $30 \mathrm{~min}$. After the TiO $\mathrm{O}_{2}$ films were cooled to room temperature, they were quickly immersed in a $0.3 \mathrm{mM}$ dye solution with different solvent and maintained in dark for $18 \mathrm{~h}$ at room temperature. The sensitized electrodes were washed with corresponding solvent and dried in air. The counter electrodes were made by dropping a solution of $\mathrm{H}_{2} \mathrm{PtCl}_{6}$ in isopropyl alcohol $(10 \mathrm{mM}, 10 \mu \mathrm{L})$ onto a conducting glass substrate (FTO, $2.2 \mathrm{~mm}$ thickness, 9 ohms $/ \mathrm{sq}, 1.25 \mathrm{~cm} \times 2.0 \mathrm{~cm}$ ) with two small holes to allow the introduction injection of the liquid electrolyte. The solution was left to spread on glass substrates and then the glass substrates were heated to $400{ }^{\circ} \mathrm{C}$ for $30 \mathrm{~min}$. For the assembly of DSCs, the sensitizer-covered $\mathrm{TiO}_{2}$ electrode and Pt-counter electrode were assembled into a sandwich type cell and sealed with a hot-melt gasket made of the ionomer Surlyn 1702 (DuPont, $25 \mu \mathrm{m}$ thickness). Finally, the hole was sealed with a Surlyn sheet (50 $\mu \mathrm{m}$ thickness) and a thin glass cover by heating. 

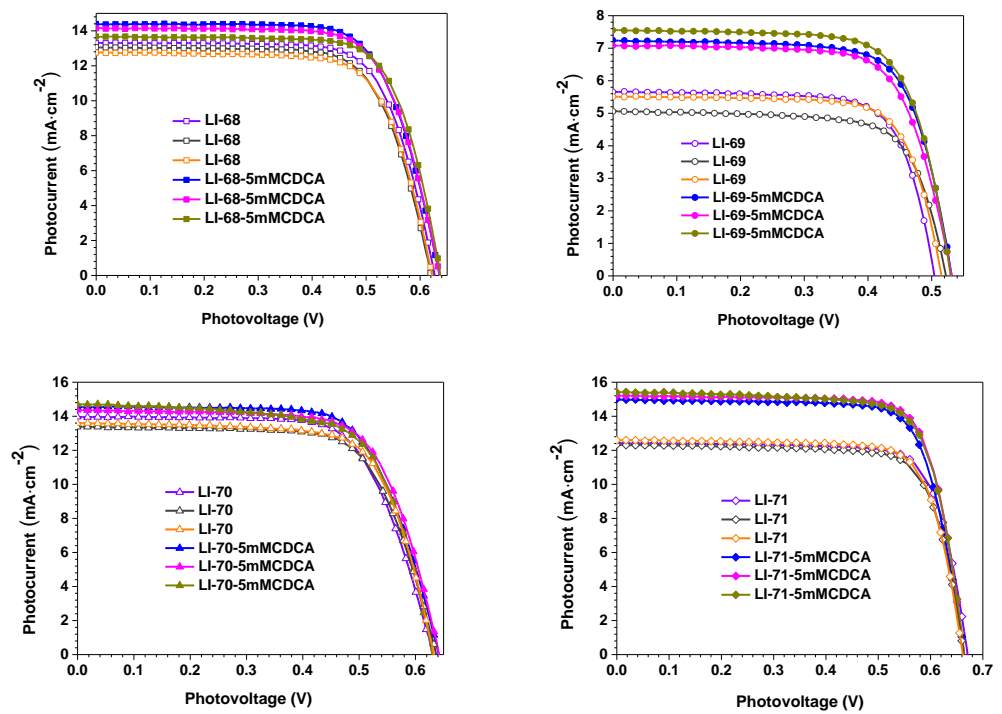

Figure S4. $J$ - $V$ characteristics of DSCs based on LI-68-LI-71 measured at simulated $100 \mathrm{~mW} \mathrm{~cm}^{-2}$ AM1.5 conditions.

\section{Experimental section}

\section{Materials}

Tetrahydrofuran (THF) was dried over and distilled from K-Na alloy under an atmosphere of dry Argon. $\mathrm{N}, \mathrm{N}$-Dimethylformamide (DMF) was dried over and distilled from $\mathrm{CaH}_{2}$ under an atmosphere of dry nitrogen. Phosphorus oxychloride $\left(\mathrm{POCl}_{3}\right)$ was freshly distilled before use. Compound $\mathbf{1},{ }^{1} \mathbf{5}^{2} \mathbf{6}^{3}$ and HexylOTPA-B ${ }^{4}$ were prepared according to literature methods. Commercially available reagents were used as received.

\section{Instrumentation}

${ }^{1} \mathrm{H}$ and ${ }^{13} \mathrm{C}$ NMR spectroscopy study was conducted with a Varian Mercury 300 spectrometer using tetramethylsilane (TMS; $\delta=0 \mathrm{ppm}$ ) as internal standard. HRMS (ESI) spectra were measured on a Finnigan LCQ advantage mass spectrometer. UV-visible spectra were obtained using a Shimadzu UV-2550 spectrometer. Electrochemical cyclic voltammetry was performed with a CHI 660 voltammetric analyzer with $\mathrm{Pt}$ disk, $\mathrm{Pt}$ plate, and $\mathrm{Ag} / \mathrm{Ag}^{+}$electrode as working electrode, counter electrode, and reference electrode, respectively, in nitrogen-purged anhydrous $\mathrm{CH}_{2} \mathrm{Cl}_{2}$ with tetrabutylammonium hexafluorophosphate $\left(\mathrm{TBAPF}_{6}\right)$ as the supporting electrolyte (scanning rate: 100 
$\mathrm{mV} / \mathrm{s})$. The ferrocene/ferrocenium redox couple was used for potential calibration.

\section{Device fabrication}

The double layer $\mathrm{TiO}_{2}$ films were prepared according to the reported procedures. Before be immersed into the dye solutions, the films were soaked in the $0.04 \mathrm{M}$ aqueous $\mathrm{TiCl}_{4}$ solution at $70{ }^{\circ} \mathrm{C}$ for $30 \mathrm{~min}$. After being washed with water and ethanol, they were heated at $500{ }^{\circ} \mathrm{C}$ for $30 \mathrm{~min}$. Subsequently, the films were dipped into a $3 \times 10^{-4} \mathrm{M} \mathrm{CH}_{3} \mathrm{CN} / \mathrm{CHCl}_{3}(\mathrm{~V} / \mathrm{V}=7 / 3)$ solution of the sensitizers for $24 \mathrm{~h}$ at room temperature. For the coadsorption, chenodeoxycholic acid (CDCA) was added. The PVP capped Pt counter electrodes were prepared through a "two-step dip coating" process, followed by heating at 325 ${ }^{\circ} \mathrm{C}$ for 10 min. The dye-loaded $\mathrm{TiO}_{2}$ electrode and Pt counter electrode were assembled into a sealed sandwich-type cell with a Surlyn sheet. The redox electrolyte, which was composed of $0.05 \mathrm{M} \mathrm{I}_{2}, 0.2 \mathrm{M}$ LiI, 0.6 M DMPII, 0.1 M GuNCS and 0.5 M 4-TBP in acetonitrile, was injected into the cell through a pre-drilled hole at the counter electrode. Finally, the hole was sealed using a Surlyn sheet and a thin glass.

\section{Photovoltaic properties measurements}

Photovoltaic performances were tested under AM $1.5 \mathrm{G}$ simulated sunlight $\left(100 \mathrm{~mW} \mathrm{~cm}^{-2}\right)$ provided by a class-AAA solar simulator (Model 11016A, Sun 3000, ABET Technologies) with a $550 \mathrm{~W}$ xenon light source. The light intensity was determined using a certificated KG-5 reference monocrystalline silicon cell system. The current-voltage $(J-V)$ curves of the DSCs were obtained by using a Keithley digital source meter (Model 2400). The electrochemical impedance spectra (EIS) measurement was measured using the Autolab PGSTAT302N electrochemical workstation under dark condition with a forward bias of $-0.7 \mathrm{~V}$ and recorded over a frequency range from $0.1 \mathrm{~Hz}$ to $100 \mathrm{KHz}$ (amplitude: $10 \mathrm{mV}$ ). Intensity-modulated photovoltage spectroscopy (IMVS) measurement was performed using the same electrochemical workstation. Incident photon-current conversion efficiency (IPCE) was recorded in a solar cell QE/IPCE measurement system equipped with a $300 \mathrm{~W}$ xenon lamp light source (Model 6258, Newport Oriel) with a cornerstone $2601 / 4 \mathrm{~m}$ monochromator. The beam 
power intensity was calibrated with a power meter (Model 1936-C, Newport). All the measurements mentioned above were taken under ambient conditions.

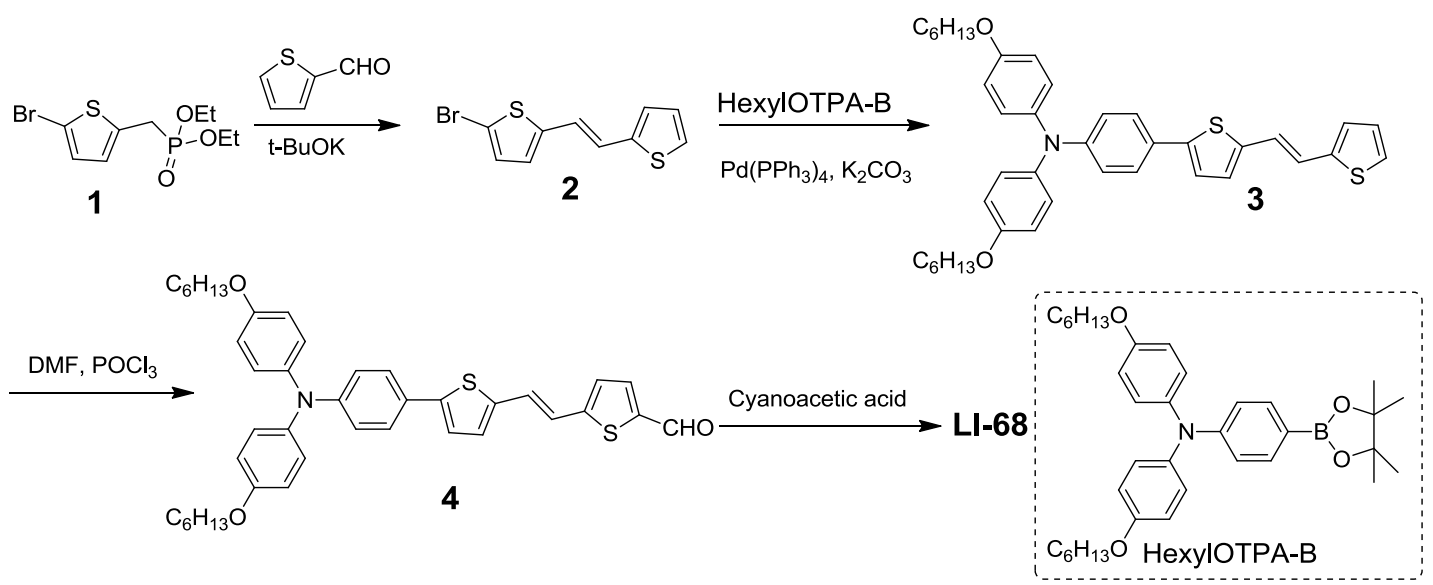

Scheme S1 Synthetic route of dye LI-68

Synthesis of 2. To a solution of compound 1 (3.76 g, $12.0 \mathrm{mmol})$ and thiophene-2-aldehyde (1.12 $\mathrm{g}, 12.0 \mathrm{mmol})$ in anhydrous THF $(24 \mathrm{~mL})$ was added $t$-BuOK $(2.24 \mathrm{~g}, 20.0 \mathrm{mmol})$ at $0{ }^{\circ} \mathrm{C}$. The resultant mixture was stirred at room temperature overnight. Then, the mixture was poured into water, neutralized with $\mathrm{HCl}(2 \mathrm{M})$ and extracted with $\mathrm{CH}_{2} \mathrm{Cl}_{2}$. The organic layer was washed with water and dried over anhydrous $\mathrm{Na}_{2} \mathrm{SO}_{4}$. After removal of the solvent, the residue was purified by column chromatography (petroleum/ $\mathrm{CHCl}_{3}, 2 / 1$ ) on silica gel to give compound 2 as a yellow solid $(2.50 \mathrm{~g}, 92 \%) .{ }^{1} \mathrm{H}$ NMR $\left(\mathrm{CDCl}_{3}, 300 \mathrm{MHz}\right) \delta(\mathrm{ppm}): 7.20(\mathrm{~s}, 1 \mathrm{H}, \mathrm{ArH}), 7.03-6.92(\mathrm{~m}, 5 \mathrm{H},-\mathrm{CH}=\mathrm{CH}-$ and $\mathrm{ArH}), 6.77$ (s, 1H, ArH).

Synthesis of 3. A solution of compound 2 (271 mg, $1.0 \mathrm{mmol})$, HexylOTPA-B (686 mg, 1.2 $\mathrm{mmol}), \mathrm{K}_{2} \mathrm{CO}_{3}(414 \mathrm{mg}, 3.0 \mathrm{mmol})$ and $\mathrm{Pd}\left(\mathrm{PPh}_{3}\right)_{4}(58 \mathrm{mg}, 0.05 \mathrm{mmol})$ in $\mathrm{THF}(24 \mathrm{~mL})$ and water $(4$ mL) was refluxed overnight. After cooling, the reaction mixture was poured into water, neutralized with $\mathrm{HCl}(2 \mathrm{M})$ and extracted with $\mathrm{CH}_{2} \mathrm{Cl}_{2}$. The organic layer was washed with water and dried over anhydrous $\mathrm{Na}_{2} \mathrm{SO}_{4}$. After removal of the solvent, the residue was purified by column chromatography (petroleum/ethyl acetate, 20/1) on silica gel to give compound $\mathbf{3}$ as an orange oil (530 mg, 83\%). ${ }^{1} \mathrm{H} \mathrm{NMR}\left(\mathrm{CDCl}_{3}, 300 \mathrm{MHz}\right) \delta(\mathrm{ppm}): 7.38(\mathrm{~d}, J=8.4 \mathrm{~Hz}, 2 \mathrm{H}, \mathrm{ArH}), 7.16(\mathrm{~s}, 1 \mathrm{H}$, ArH), 7.05-6.95 (m, 11H, ArH and -CH=CH-), 6.91 (s, br, 1H, ArH), 6.83 (d, J=8.4 H, 4H, ArH), 
$3.93\left(\mathrm{t}, J=6.0 \mathrm{~Hz}, 4 \mathrm{H},-\mathrm{O}-\mathrm{CH}_{2}-\right), 1.80-1.73\left(\mathrm{~m}, 4 \mathrm{H},-\mathrm{CH}_{2^{-}}\right), 1.46\left(\mathrm{~s}, \mathrm{br}, 4 \mathrm{H},-\mathrm{CH}_{2^{-}}\right), 1.36(\mathrm{~s}, \mathrm{br}, 8 \mathrm{H}$, $\left.-\mathrm{CH}_{2-}\right), 0.91\left(\mathrm{t}, J=6.6 \mathrm{~Hz}, 6 \mathrm{H},-\mathrm{CH}_{3}\right)$.

Synthesis of 4. A mixture of dry DMF $(0.13 \mathrm{~mL}, 1.7 \mathrm{mmol})$ and $\mathrm{POCl}_{3}(0.12 \mathrm{~mL}, 1.2 \mathrm{mmol})$ was stirred at $0{ }^{\circ} \mathrm{C}$ for $30 \mathrm{~min}$. Then, a solution of compound $\mathbf{3}(530 \mathrm{mg}, 0.83 \mathrm{mmol})$ in 1,2-dichloroethane $(15 \mathrm{~mL})$ was added to the above mixture. The reaction was refluxed for $4 \mathrm{~h}$. After cooling, the resultant solution was slowly added into a solution of $\mathrm{Na}_{2} \mathrm{CO}_{3}(2 \mathrm{M})$, stirred for 1 $\mathrm{h}$ and extracted with $\mathrm{CH}_{2} \mathrm{Cl}_{2}$. The organic layer was washed with water and dried over anhydrous $\mathrm{Na}_{2} \mathrm{SO}_{4}$. After removal of the solvent, the residue was purified by column chromatography (petroleum/ $\left.\mathrm{CH}_{2} \mathrm{Cl}_{2}, 10 / 1\right)$ on silica gel to give compound 4 as an orange oil $(390 \mathrm{mg}, 71 \%) .{ }^{1} \mathrm{H}$ $\operatorname{NMR}\left(\mathrm{CDCl}_{3}, 300 \mathrm{MHz}\right) \delta(\mathrm{ppm}): 9.83(\mathrm{~s}, 1 \mathrm{H},-\mathrm{CHO}), 7.64(\mathrm{~d}, J=3.9 \mathrm{~Hz}, 1 \mathrm{H}, \mathrm{ArH}), 7.39(\mathrm{~d}, J=$ $8.4 \mathrm{~Hz}, 2 \mathrm{H}, \mathrm{ArH}), 7.23$ (d, $J=18.0 \mathrm{~Hz}, 1 \mathrm{H},-\mathrm{CH}=\mathrm{CH}-), 7.09-7.05$ (m, 7H, ArH), 6.98-6.89 (m, 3H, ArH and $-\mathrm{CH}=\mathrm{CH}-), 6.84(\mathrm{~d}, J=8.7 \mathrm{~Hz}, 4 \mathrm{H}, \mathrm{ArH}), 3.94\left(\mathrm{t}, J=6.3 \mathrm{~Hz}, 4 \mathrm{H},-\mathrm{O}-\mathrm{CH}_{2}-\right), 1.80-1.76(\mathrm{~m}$, 4H, $-\mathrm{CH}_{2^{-}}$), 1.46 (s, br, 4H, $\left.-\mathrm{CH}_{2^{-}}\right), 1.34$ (s, br, 8H, $\left.-\mathrm{CH}_{2^{-}}\right), 0.91$ (s, br, 6H, $-\mathrm{CH}_{3}$ ).

Synthesis of dye LI-68. A solution of compound 4 (300 mg, $0.45 \mathrm{mmol})$, ammonium acetate (100 $\mathrm{mg}, 1.30 \mathrm{mmol})$ and cyanoacetic acid $(307 \mathrm{mg}, 3.61 \mathrm{mmol})$ in acetic acid $(10 \mathrm{~mL})$ was refluxed overnight. After cooling, the reaction mixture was poured into water, and the solid was precipitated from the solution. The crude product was separated by filtration, then purified by column chromatography $\left(\mathrm{CH}_{2} \mathrm{Cl}_{2} /\right.$ methanol, 20/1) on silica gel to give dye $\mathbf{L I - 6 8}$ as a black solid (157 mg, 48\%). ${ }^{1} \mathrm{H}$ NMR $\left(\mathrm{DMSO}-d_{6}, 300 \mathrm{MHz}\right) \delta(\mathrm{ppm}): 8.28(\mathrm{~s}, 1 \mathrm{H},-\mathrm{CH}=), 7.79(\mathrm{~s}, 1 \mathrm{H}, \mathrm{ArH}), 7.47(\mathrm{~d}, J=8.7 \mathrm{~Hz}, 2 \mathrm{H}, \mathrm{ArH})$, 7.40-7.31 (m, 4H, ArH and -CH=CH-), $7.16(\mathrm{~d}, J=16.2 \mathrm{~Hz}, 1 \mathrm{H},-\mathrm{CH}=\mathrm{CH}-), 7.03$ (d, $J=8.4 \mathrm{~Hz}, 4 \mathrm{H}$, $\operatorname{ArH}), 6.93(\mathrm{~d}, J=8.4 \mathrm{~Hz}, 4 \mathrm{H}, \mathrm{ArH}), 6.75(\mathrm{~d}, J=8.1 \mathrm{~Hz}, 2 \mathrm{H}, \mathrm{ArH}), 3.93\left(\mathrm{t}, J=6.0 \mathrm{~Hz}, 4 \mathrm{H},-\mathrm{OCH}_{2^{-}}\right)$, 1.69 (s, br, 4H, $\left.-\mathrm{CH}_{2}-\right), 1.40\left(\mathrm{~s}, \mathrm{br}, 4 \mathrm{H},-\mathrm{CH}_{2}-\right), 1.30\left(\mathrm{~s}, \mathrm{br}, 8 \mathrm{H},-\mathrm{CH}_{2}-\right), 0.88\left(\mathrm{~s}, \mathrm{br}, 6 \mathrm{H},-\mathrm{CH}_{3}\right) .{ }^{13} \mathrm{C} \mathrm{NMR}$ $\left(\mathrm{DMSO}-d_{6}, 100 \mathrm{MHz}\right) \delta(\mathrm{ppm}): 164.72,156.37,152.63,149.25,146.82,145.77,141.86,140.25,139.85$, $134.79,131.77,128.06,127.76,127.08,126.89,125.57,123.75,120.01,119.79,117.62,116.24,98.73$, 68.52, 32.00, 29.69, 26.20, 23.04, 14.80. HRMS (ESI, $m / z)$ : [M-1] calcd for $\mathrm{C}_{44} \mathrm{H}_{45} \mathrm{~N}_{2} \mathrm{O}_{4} \mathrm{~S}_{2}, 729.2821$, 
Found, 729.2816.

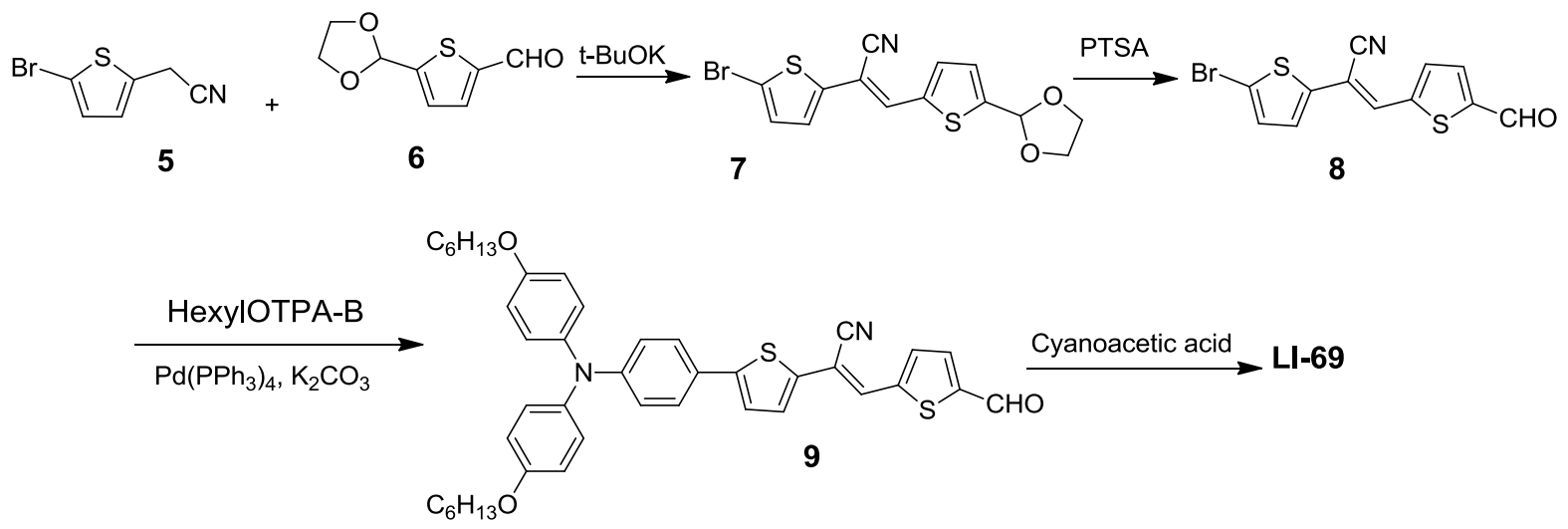

Scheme S2 Synthetic route of dye LI-69

Synthesis of 7. To a solution of compound $5(1.21 \mathrm{~g}, 6.0 \mathrm{mmol})$ and compound $6(1.17 \mathrm{~g}, 6.4 \mathrm{mmol})$ was added $t$-BuOK $(1.68 \mathrm{~g}, 15.0 \mathrm{mmol})$ in batches. The resultant mixture was stirred at room temperature for $2 \mathrm{~h}$. Then, the crude product was separated by filtration, washed with water and ethanol, then further purified by recrystallization in petroleum to give compound 7 as a yellow solid (1.32 g, 60\%). ${ }^{1} \mathrm{H}$ NMR $\left(\mathrm{CDCl}_{3}, 300 \mathrm{MHz}\right) \delta(\mathrm{ppm}): 7.48(\mathrm{~d}, J=3.6 \mathrm{~Hz}, 1 \mathrm{H}, \mathrm{ArH}), 7.29(\mathrm{~s}, 1 \mathrm{H},-\mathrm{CH}=), 7.16(\mathrm{~d}$, $J=3.9 \mathrm{~Hz}, 1 \mathrm{H}, \mathrm{ArH}), 7.09(\mathrm{~d}, J=3.9 \mathrm{~Hz}, 1 \mathrm{H}, \mathrm{ArH}), 7.03(\mathrm{~d}, J=3.9 \mathrm{~Hz}, 1 \mathrm{H}, \operatorname{ArH}), 6.13(\mathrm{~s}, 1 \mathrm{H}, \operatorname{ArH})$, 4.18-4.02 (m, 4H, $\left.-\mathrm{CH}_{2-}\right)$.

Synthesis of 8. To a solution of compound 7 (500 mg, $1.36 \mathrm{mmol})$ in THF (40 mL) and water (4 mL) was added $p$-toluene sulphonic acid (PTSA, $50 \mathrm{mg}, 0.29 \mathrm{mmol}$ ). The resultant mixture was stirred at room temperature overnight. The crude product was separated by filtration, then washed with water and ethanol to give compound 8 as a yellow solid (380 mg, 86\%). ${ }^{1} \mathrm{H} \mathrm{NMR}\left(\mathrm{CDCl}_{3}, 300 \mathrm{MHz}\right) \delta(\mathrm{ppm}): 9.96$ (s, 1H, -CHO), 7.77 (d, J=3.9 Hz, 1H, ArH), 7.73 (d, J=3.3 Hz, 1H, ArH), 7.30 (s, 1H, -CH=), 7.19 (d, $J=4.2 \mathrm{~Hz}, 1 \mathrm{H}, \mathrm{ArH}), 7.08(\mathrm{~d}, J=3.9 \mathrm{~Hz}, 1 \mathrm{H}, \mathrm{ArH})$.

Synthesis of 9. Compound 9 (312 mg, 91\%) was obtained as a red solid by the similar procedure with that of compound 3. ${ }^{1} \mathrm{H} \mathrm{NMR}\left(\mathrm{CDCl}_{3}, 300 \mathrm{MHz}\right) \delta(\mathrm{ppm}): 9.94(\mathrm{~s}, 1 \mathrm{H},-\mathrm{CHO}), 7.75(\mathrm{~d}, J=3.9 \mathrm{~Hz}, 1 \mathrm{H}$, $\operatorname{ArH}), 7.71(\mathrm{~d}, J=4.5 \mathrm{~Hz}, 1 \mathrm{H}, \mathrm{ArH}), 7.40-7.33(\mathrm{~m}, 4 \mathrm{H}, \mathrm{ArH}$ and $-\mathrm{CH}=), 7.14(\mathrm{~d}, J=3.6 \mathrm{~Hz}, 1 \mathrm{H}, \mathrm{ArH})$, $7.07(\mathrm{~d}, J=9.0 \mathrm{~Hz}, 4 \mathrm{H}, \mathrm{ArH}), 6.91-6.83(\mathrm{~m}, 6 \mathrm{H}, \mathrm{ArH}), 3.94\left(\mathrm{t}, J=6.6 \mathrm{~Hz}, 4 \mathrm{H},-\mathrm{O}-\mathrm{CH}_{2}-\right), 1.81-1.73(\mathrm{~m}$, 
4H, $-\mathrm{CH}_{2-}$ ), 1.47 (s, br, 4H, - $\mathrm{CH}_{2^{-}}$), 1.35 (s, br, 8H, $\left.-\mathrm{CH}_{2}-\right), 0.91$ (t, J = 6.3 Hz, 6H, - $\mathrm{CH}_{3}$ ).

Synthesis of dye LI-69. Dye LI-69 was synthesized by the similar procedure with that of dye LI-68. After the separation by column chromatography, the obtained product was further purified by recrystallization in acetic acid to give dye $\mathbf{L I - 6 9}$ as a black solid (105 mg, 38\%). ${ }^{1} \mathrm{H}$ NMR $\left(\mathrm{CDCl}_{3}\right.$, 300MHz) $\delta(\mathrm{ppm}): 8.50(\mathrm{~s}, 1 \mathrm{H},=\mathrm{CH}-), 8.03-8.01(\mathrm{~m}, 2 \mathrm{H}, \mathrm{ArH}$ and $-\mathrm{CH}=\mathrm{CH}-), 7.83(\mathrm{~d}, J=4.5 \mathrm{~Hz}, 1 \mathrm{H}$, ArH), $7.50(\mathrm{~d}, J=8.1 \mathrm{~Hz}, 2 \mathrm{H}, \operatorname{ArH}), 7.44-7.42(\mathrm{~m}, 2 \mathrm{H}, \mathrm{ArH}), 7.06(\mathrm{~d}, J=8.4 \mathrm{~Hz}, 4 \mathrm{H}, \operatorname{ArH}), 6.92(\mathrm{~d}, J=$ $8.7 \mathrm{~Hz}, 4 \mathrm{H}, \mathrm{ArH}), 6.75(\mathrm{~d}, J=8.7 \mathrm{~Hz}, 2 \mathrm{H}, \mathrm{ArH}), 3.94\left(\mathrm{t}, J=6.2 \mathrm{~Hz}, 4 \mathrm{H},-\mathrm{O}-\mathrm{CH}_{2^{-}}\right), 1.70$ (s, br, 4H, $-\mathrm{CH}_{2^{-}}$), 1.41 (s, br, 4H, $-\mathrm{CH}_{2^{-}}$), $1.30\left(\mathrm{~s}, \mathrm{br}, 8 \mathrm{H},-\mathrm{CH}_{2^{-}}\right), 0.88\left(\mathrm{~s}, \mathrm{br}, 6 \mathrm{H},-\mathrm{CH}_{3}\right) .{ }^{13} \mathrm{C}$ NMR (DMSO- $d_{6}, 100$ $\mathrm{MHz}) \delta(\mathrm{ppm}): 164.06,156.51,149.56,147.28,146.11,145.73,139.99,139.70,138.98,136.10,134.02$, $130.50,130.18,127.92,127.09,124.58,123.99,119.10,117.02,116.29,105.93,102.02,68.41,31.85$, 29.52, 26.12, 22.98, 14.77. HRMS (ESI, m/z): [M-1] calcd for $\mathrm{C}_{45} \mathrm{H}_{44} \mathrm{~N}_{3} \mathrm{O}_{4} \mathrm{~S}_{2}, 754.2773$; Found, 754.2786.

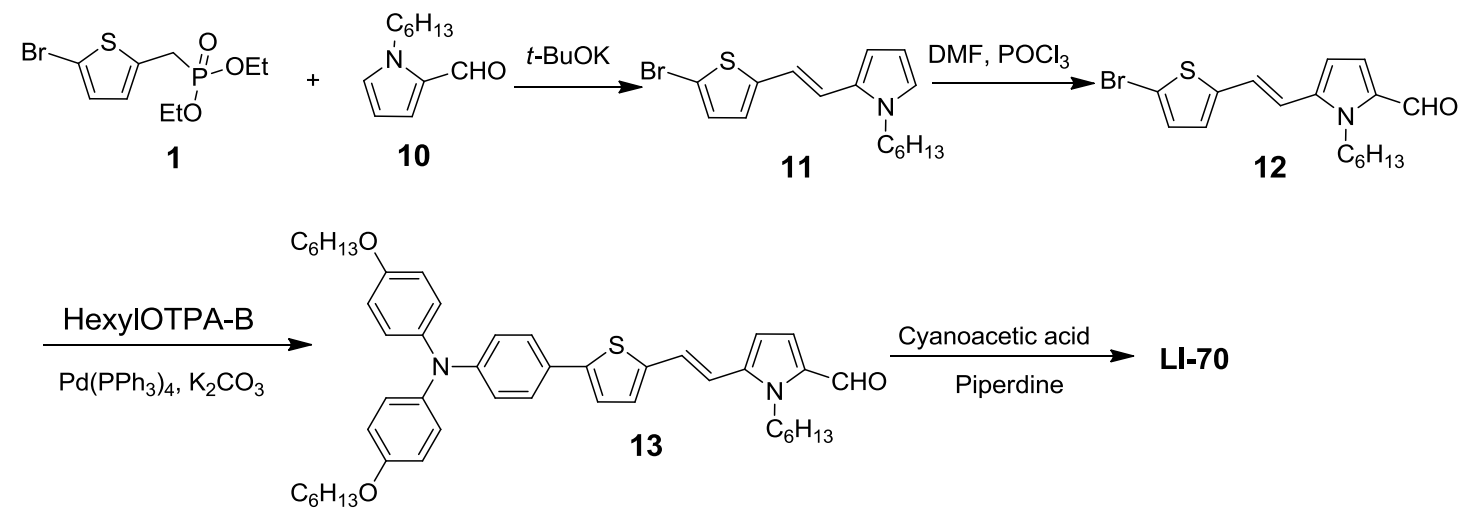

Scheme S3 Synthetic route of dye LI-70

Synthesis of 11. Compound $11(2.57 \mathrm{~g}, 76 \%)$ was obtained as a yellow oil by the similar procedures of compound 2. ${ }^{1} \mathrm{H} \mathrm{NMR}\left(\mathrm{CDCl}_{3}, 300 \mathrm{MHz}\right) \delta(\mathrm{ppm}): 6.92(\mathrm{~d}, J=3.9 \mathrm{~Hz}, 1 \mathrm{H}, \mathrm{ArH}), 6.85(\mathrm{~d}, J=15.9$ $\mathrm{Hz}, 1 \mathrm{H},-\mathrm{CH}=\mathrm{CH}-), 6.70-6.62(\mathrm{~m}, 3 \mathrm{H}, \mathrm{ArH}$ and $-\mathrm{CH}=\mathrm{CH}-), 6.44(\mathrm{~s}, 1 \mathrm{H}, \mathrm{ArH}), 6.14$ (s, 1H, ArH), $3.91(\mathrm{t}$, $\left.J=7.2 \mathrm{~Hz}, 2 \mathrm{H},-\mathrm{N}-\mathrm{CH}_{2^{-}}\right), 1.72\left(\mathrm{~s}, \mathrm{br}, 2 \mathrm{H},-\mathrm{CH}_{2}-\right), 1.30$ (s, br, 6H, $\left.-\mathrm{CH}_{2^{-}}\right), 0.88\left(\mathrm{~s}, \mathrm{br}, 3 \mathrm{H},-\mathrm{CH}_{3}\right)$.

Synthesis of 12. A mixture of dry DMF $(0.17 \mathrm{~mL}, 2.20 \mathrm{mmol})$ and $\mathrm{POCl}_{3}(0.15 \mathrm{~mL}, 1.61 \mathrm{mmol})$ was stirred at $0{ }^{\circ} \mathrm{C}$ for $30 \mathrm{~min}$. Then, a solution of compound $\mathbf{1 1}(365 \mathrm{mg}, 1.10 \mathrm{mmol})$ in 1,2-dichloroethane 
$(15 \mathrm{~mL})$ was added to the above mixture, and the solution was stirred at room temperature overnight. The resultant solution was slowly added into a solution of $\mathrm{Na}_{2} \mathrm{CO}_{3}(2 \mathrm{M})$, stirred for 30 min and extracted with $\mathrm{CH}_{2} \mathrm{Cl}_{2}$. The organic layer was washed with water and dried over anhydrous $\mathrm{Na}_{2} \mathrm{SO}_{4}$. After removal of the solvent, the residue was purified by column chromatography (petroleum/ethyl acetate, 30/1) on silica gel to give compound 12 as a yellow oil $(233 \mathrm{mg}, 59 \%) .{ }^{1} \mathrm{H} \mathrm{NMR}\left(\mathrm{CDCl}_{3}, 300\right.$ MHz) $\delta(\mathrm{ppm}): 9.46(\mathrm{~s}, 1 \mathrm{H},-\mathrm{CHO}), 7.10(\mathrm{~d}, J=15.9 \mathrm{~Hz}, 1 \mathrm{H},-\mathrm{CH}=\mathrm{CH}-), 6.97$ (d, $J=3.9 \mathrm{~Hz}, 1 \mathrm{H}, \operatorname{ArH})$, $6.90(\mathrm{~d}, J=4.2 \mathrm{~Hz}, 1 \mathrm{H}, \mathrm{ArH}), 6.83(\mathrm{~d}, J=3.6 \mathrm{~Hz}, 1 \mathrm{H}, \mathrm{ArH}), 6.65(\mathrm{~d}, J=15.9 \mathrm{~Hz}, 1 \mathrm{H},-\mathrm{CH}=\mathrm{CH}-), 6.51$ (d, $J=4.2 \mathrm{~Hz}, 1 \mathrm{H}, \mathrm{ArH}), 4.41\left(\mathrm{t}, J=7.2 \mathrm{~Hz}, 2 \mathrm{H},-\mathrm{N}-\mathrm{CH}_{2^{-}}\right), 1.70\left(\mathrm{~s}, \mathrm{br}, 2 \mathrm{H},-\mathrm{CH}_{2}-\right), 1.31(\mathrm{~s}, \mathrm{br}, 6 \mathrm{H}$, $\left.-\mathrm{CH}_{2^{-}}\right), 0.88\left(\mathrm{~s}, \mathrm{br}, 3 \mathrm{H},-\mathrm{CH}_{3}\right)$.

Synthesis of 13. Compound $13(390 \mathrm{mg}, 84 \%)$ was obtained as an organge oil by the similar procedure of compound 3. ${ }^{1} \mathrm{H}$ NMR $\left(\mathrm{CDCl}_{3}, 300 \mathrm{MHz}\right) \delta(\mathrm{ppm}): 9.45(\mathrm{~s}, 1 \mathrm{H},-\mathrm{CHO}), 7.40(\mathrm{~d}, J=8.4$ $\mathrm{Hz}, 2 \mathrm{H}, \mathrm{ArH}), 7.19$ (d, $J=15.6 \mathrm{~Hz}, 1 \mathrm{H},-\mathrm{CH}=\mathrm{CH}-), 7.08-7.01(\mathrm{~m}, 6 \mathrm{H}, \mathrm{ArH}), 6.92-6.82(\mathrm{~m}, 7 \mathrm{H}, \mathrm{ArH})$, $6.71(\mathrm{~d}, J=15.6 \mathrm{~Hz}, 1 \mathrm{H},-\mathrm{CH}=\mathrm{CH}-), 6.52(\mathrm{~d}, J=4.2 \mathrm{~Hz}, 1 \mathrm{H}, \mathrm{ArH}), 4.41\left(\mathrm{t}, J=7.2 \mathrm{~Hz}, 2 \mathrm{H},-\mathrm{N}^{-} \mathrm{CH}_{2^{-}}\right)$, $3.94\left(\mathrm{t}, J=6.6 \mathrm{~Hz}, 4 \mathrm{H},-\mathrm{O}-\mathrm{CH}_{2}-\right), 1.80-1.73\left(\mathrm{~m}, 6 \mathrm{H},-\mathrm{CH}_{2^{-}}\right), 1.47\left(\mathrm{~s}, \mathrm{br}, 4 \mathrm{H},-\mathrm{CH}_{2^{-}}\right), 1.34(\mathrm{~s}, \mathrm{br}, 14 \mathrm{H}$, $\left.-\mathrm{CH}_{2^{-}}\right), 0.91-0.88\left(\mathrm{~m}, 9 \mathrm{H},-\mathrm{CH}_{3}\right)$.

Synthesis of dye LI-70. A solution of compound 13 (340 mg, $0.46 \mathrm{mmol}$ ), cyanoacetic acid (60 mg, $0.70 \mathrm{mmol})$ and piperdine $(10 \mu \mathrm{L})$ in THF $(5 \mathrm{~mL})$ and acetonitrile $(10 \mathrm{~mL})$ was refluxed overnight. After cooling, the reaction mixture was poured into $\mathrm{HCl}(1 \mathrm{M}, 100 \mathrm{~mL})$, stirred for $1 \mathrm{~h}$ and extracted with $\mathrm{CH}_{2} \mathrm{Cl}_{2}$. The organic layer was washed with water and dried over anhydrous $\mathrm{Na}_{2} \mathrm{SO}_{4}$. Then, the solvent was removed, and the residue was purified by column chromatography $\left(\mathrm{CH}_{2} \mathrm{Cl}_{2} / \mathrm{methanol}, 20 / 1\right)$ on silica gel as a black solid (226 mg, 66\%). ${ }^{1} \mathrm{H}$ NMR (DMSO- $\left.d_{6}, 300 \mathrm{MHz}\right) \delta(\mathrm{ppm}): 7.97(\mathrm{~s}, 1 \mathrm{H}$, $-\mathrm{CH}=), 7.56(\mathrm{~d}, J=3.9 \mathrm{~Hz}, 1 \mathrm{H}, \mathrm{ArH}), 7.51-7.42(\mathrm{~m}, 3 \mathrm{H}, \mathrm{ArH}$ and $-\mathrm{CH}=\mathrm{CH}-), 7.28-7.26(\mathrm{~m}, 2 \mathrm{H}, \mathrm{ArH})$, 7.01-6.86 (m, 10H, ArH and -CH=CH-), $6.72(\mathrm{~d}, J=8.4 \mathrm{~Hz}, 2 \mathrm{H}, \mathrm{ArH}), 4.23\left(\mathrm{~s}, \mathrm{br}, 2 \mathrm{H},-\mathrm{N}-\mathrm{CH}_{2^{-}}\right), 3.90$ (s, br, 4H, -O- $\left.\mathrm{CH}_{2^{-}}\right), 1.66-1.56\left(\mathrm{~m}, 4 \mathrm{H},-\mathrm{CH}_{2^{-}}\right), 1.38-1.22\left(\mathrm{~m}, 20 \mathrm{H},-\mathrm{CH}_{2^{-}}\right), 0.85\left(\mathrm{~s}, \mathrm{br}, 9 \mathrm{H},-\mathrm{CH}_{3}\right) .{ }^{13} \mathrm{C}$ NMR $\left(\mathrm{CDCl}_{3}, 100 \mathrm{MHz}\right) \delta(\mathrm{ppm}): 170.23,155.85,148.90,145.69,142.03,140.11,139.55,138.25$, 
$130.01,128.20,127.43,126.94,126.39,125.34,122.39,121.96,119.86,117.63,115.36,112.87,111.56$, $88.87,68.30,43.28,31.64,31.54,31.28,29.35,26.35,25.80,22.66,22.52,14.10,14.03$. HRMS (ESI, m/z): [M-1] calcd for $\mathrm{C}_{50} \mathrm{H}_{58} \mathrm{~N}_{3} \mathrm{O}_{4} \mathrm{~S}, 796.4148$; Found,796.4139.

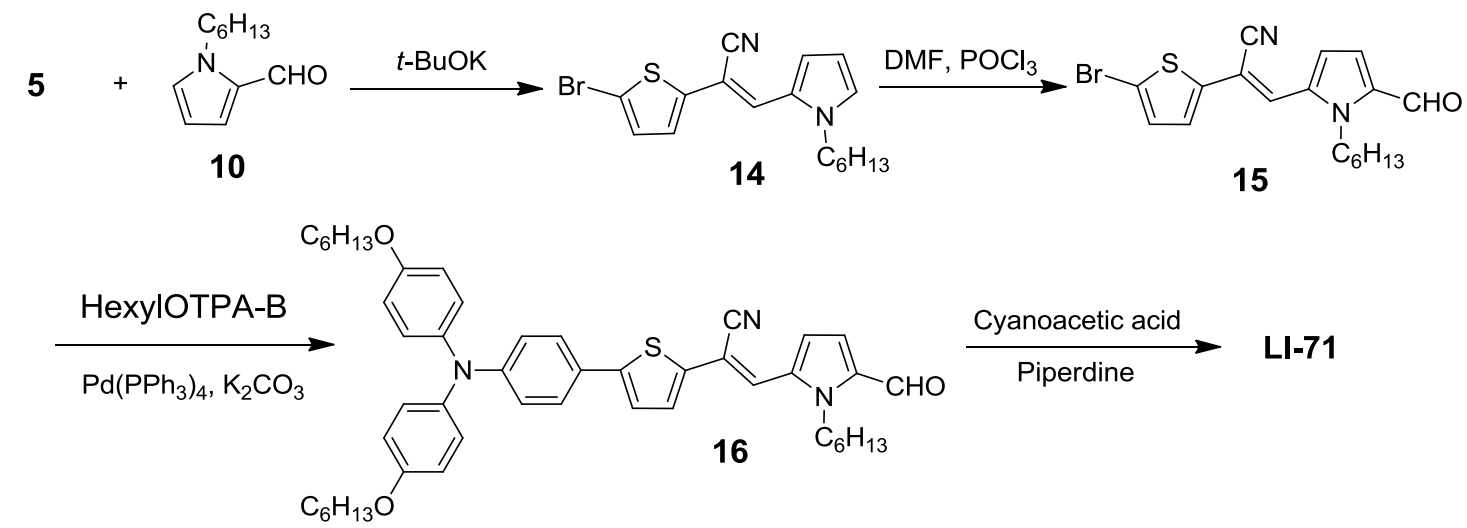

Scheme S4 Synthetic route of dye LI-71

Synthesis of 14. To a solution of compound $5(5.09 \mathrm{~g}, 25.1 \mathrm{mmol})$ and compound 10 (3.74 g, 20.9 mmol) in ethanol $(40 \mathrm{~mL})$ was added $t$-BuOK $(4.50 \mathrm{~g}, 40.2 \mathrm{mmol})$ in batches. The reaction mixture was stirred at $40{ }^{\circ} \mathrm{C}$ overnight. After cooling, the mixture was poured into water and extracted with $\mathrm{CH}_{2} \mathrm{Cl}_{2}$. The organic layer was washed with water and dried over anhydrous $\mathrm{Na}_{2} \mathrm{SO}_{4}$. Then, the solvent was removed, and the residue was purified by column chromatography (petroleum/ethyl acetate, 50/1) on silica gel to give compound 14 as a yellow oil $(5.0 \mathrm{~g}, 58 \%) .{ }^{1} \mathrm{H} \mathrm{NMR}\left(\mathrm{CDCl}_{3}, 300 \mathrm{MHz}\right) \delta(\mathrm{ppm}): 7.41$ $(\mathrm{d}, J=3.3 \mathrm{~Hz}, 1 \mathrm{H}, \mathrm{ArH}), 7.04(\mathrm{~s}, 1 \mathrm{H},-\mathrm{CH}=), 7.00(\mathrm{~s}, 2 \mathrm{H}, \mathrm{ArH}), 6.86(\mathrm{~s}, 1 \mathrm{H}, \operatorname{ArH}), 6.30(\mathrm{t}, J=3.3 \mathrm{~Hz}$, $1 \mathrm{H}, \mathrm{ArH}), 3.96\left(\mathrm{t}, J=7.2 \mathrm{~Hz}, 2 \mathrm{H},-\mathrm{N}-\mathrm{CH}_{2^{-}}\right), 1.73\left(\mathrm{~s}, \mathrm{br}, 2 \mathrm{H},-\mathrm{CH}_{2^{-}}\right), 1.30\left(\mathrm{~s}, \mathrm{br}, 4 \mathrm{H},-\mathrm{CH}_{2^{-}}\right), 0.89(\mathrm{t}, J=$ $\left.6.0 \mathrm{~Hz}, 3 \mathrm{H},-\mathrm{CH}_{3}\right)$.

Synthesis of 15. Compound 15 (3.20 g, 59\%) was obtained as a yellow solid by the similar procedure of compound 12. ${ }^{1} \mathrm{H} \mathrm{NMR}\left(\mathrm{CDCl}_{3}, 300 \mathrm{MHz}\right) \delta(\mathrm{ppm}): 9.63(\mathrm{~s}, 1 \mathrm{H},-\mathrm{CHO}), 7.38(\mathrm{~d}, J=4.2 \mathrm{~Hz}, 1 \mathrm{H}$, ArH), $7.15(\mathrm{~d}, J=3.9 \mathrm{~Hz}, 1 \mathrm{H}, \mathrm{ArH}), 7.08-7.06(\mathrm{~m}, 2 \mathrm{H}, \mathrm{ArH}$ and $-\mathrm{CH}=), 7.02(\mathrm{~d}, J=4.2 \mathrm{~Hz}, 1 \mathrm{H}, \mathrm{ArH})$, $4.46\left(\mathrm{t}, J=7.5 \mathrm{~Hz}, 2 \mathrm{H},-\mathrm{N}-\mathrm{CH}_{2^{-}}\right), 1.70\left(\mathrm{~s}, \mathrm{br}, 2 \mathrm{H},-\mathrm{CH}_{2^{-}}\right), 1.32$ (s, br, 4H, $-\mathrm{CH}_{2^{-}}$), 0.89 (s, br, 3H, $\left.-\mathrm{CH}_{3}\right)$.

Synthesis of 16. Compound $16(170 \mathrm{mg}, 88 \%)$ was obtained as a yellow solid by the similar procedure of compound 3. ${ }^{1} \mathrm{H}$ NMR $\left(\mathrm{CDCl}_{3}, 300 \mathrm{MHz}\right) \delta(\mathrm{ppm})$ : $9.60(\mathrm{~s}, 1 \mathrm{H},-\mathrm{CHO}), 7.40-7.32(\mathrm{~m}, 4 \mathrm{H}$, 
ArH and $-\mathrm{CH}=), 7.14-7.01(\mathrm{~m}, 7 \mathrm{H}, \mathrm{ArH}), 6.92-6.83(\mathrm{~m}, 6 \mathrm{H}, \mathrm{ArH}), 4.48\left(\mathrm{t}, J=6.6 \mathrm{~Hz}, 2 \mathrm{H},-\mathrm{N}_{-} \mathrm{CH}_{2^{-}}\right)$,

$3.94\left(\mathrm{t}, J=6.0 \mathrm{~Hz}, 4 \mathrm{H},-\mathrm{CH}_{2^{-}}\right), 1.81-1.73\left(\mathrm{~m}, 6 \mathrm{H},-\mathrm{CH}_{2^{-}}\right), 1.46\left(\mathrm{~s}, \mathrm{br}, 4 \mathrm{H},-\mathrm{CH}_{2^{-}}\right), 1.34(\mathrm{~s}, \mathrm{br}, 14 \mathrm{H}$, $\left.-\mathrm{CH}_{2-}\right), 0.91\left(\mathrm{~s}, \mathrm{br}, 9 \mathrm{H},-\mathrm{CH}_{3}\right)$.

Synthesis of dye LI-71. Dye LI-71 (178 mg, 94\%) was obtained as a black solid by the similar

procedure of dye LI-70. ${ }^{1} \mathrm{H}$ NMR (DMSO-d $\left.6,300 \mathrm{MHz}\right) \delta(\mathrm{ppm}): 8.09$ (s, 1H, -CH=), 7.86 (s, 1H, ArH), $7.53(\mathrm{~s}, 1 \mathrm{H},-\mathrm{CH}=), 7.38(\mathrm{~d}, J=8.1 \mathrm{~Hz}, 2 \mathrm{H}, \mathrm{ArH}), 7.32(\mathrm{~s}, 1 \mathrm{H}, \mathrm{ArH}), 7.13-7.05$ (m, 6H, ArH), 6.91-6.83 (m, 6H, ArH), $4.14\left(\mathrm{~s}, \mathrm{br}, 2 \mathrm{H},-\mathrm{N}-\mathrm{CH}_{2}-\right), 3.94\left(\mathrm{t}, J=6.0 \mathrm{~Hz}, 4 \mathrm{H},-\mathrm{CH}_{2}-\right), 1.81-1.73\left(\mathrm{~m}, 6 \mathrm{H},-\mathrm{CH}_{2}-\right), 1.46$ (s, br, 4H, $\left.-\mathrm{CH}_{2}-\right), 1.34$ (s, br, $\left.14 \mathrm{H},-\mathrm{CH}_{2}-\right), 0.91\left(\mathrm{~s}, \mathrm{br}, 9 \mathrm{H},-\mathrm{CH}_{3}\right) .{ }^{13} \mathrm{C} \mathrm{NMR}\left(\mathrm{CDCl}_{3}, 100 \mathrm{MHz}\right) \delta(\mathrm{ppm})$ : $169.21,156.06,149.41,147.04,139.92,136.42,135.95,129.90,129.69,127.18,126.97,126,62,124.44$, $122.64,121.39,119.57,116.71,116.46,115.67,115.46,107.01,68.36,43.61,31.80,31.37,29.85,29.44$, 26.49, 25.91, 22.78, 22.63, 14.23, 14.10. HRMS (ESI, $m / z$ ): [M-1] $]^{-}$calcd for $\mathrm{C}_{51} \mathrm{H}_{57} \mathrm{~N}_{4} \mathrm{O}_{4} \mathrm{~S}, 821.4101$; Found, 821.4095.

\section{Reference}

1. Chung, D. S.; Lee, S. J.; Park, J. W.; Choi, D. B.; Lee, D. H.; Park, J. W.; Shin, S. C.; Kim, Y. H.; Kwon S. K.; Park, C. E. High Performance Amorphous Polymeric Thin-Film Transistors Based on Poly[(1,2-bis-(2'-thienyl)vinyl-5',5'’-diyl)-alt-(9,9-dioctylfluorene-2,7-diyl] Semiconductors. Chem. Mater. 2008, 20, 3450-3456.

2. Chung, J. W.; Yang, H.; Singh, B.; Moon, H.; An, B. K.; Lee, S. Y.; Park, S. Y. Single-Crystalline Organic Nanowires with Large Mobility and Strong Fluorescence Emission: a Conductive-AFM and Space-Charge-Limited-Current Study. J. Mater. Chem. 2009, 19, 5920-5925.

3. Zhang, L.; Clark, R. J.; Zhu, L. A Heteroditopic Fluoroionophoric Platform for Constructing Fluorescent Probes with Large Dynamic Ranges for Zinc Ions. Chem. Eur. J. 2008, 14, 2894-2903.

4. Li, R.; Liu, J.; Cai, N.; Zhang, M.; Wang, P. Synchronously Reduced Surface States, Charge Recombination, and Light Absorption Length for High-Performance Organic Dye-Sensitized Solar Cells. J. Phys. Chem. B 2010, 114, 4461-4464. 